\title{
Laser Management Of Introral Soft Tissue Lesions - A Review Of Literature
}

\author{
Reshma J Abraham ${ }^{1}$, Arathy S Lankupalli ${ }^{2}$ \\ I (BDS CRRI, Department of Oral Medicine, Saveetha Dental College,India) \\ ${ }_{2}^{2}$ (Senior Lecturer, Department of Oral medicine, Saveetha Dental College, India)
}

\begin{abstract}
In 1917, the theory of "stimulated emission" was put forward by Albert Einstein, stating that photons could "stimulate" the emission of another photon that would possess identical properties to the first. Townes and Schawlow worked on a study together that led to the development of LASER(Light Amplification by Stimulated Emission of Radiation). The initial developments of laser research were started in the USA and Soviet Union in the year 1958. In the year 1960, Theodore Maiman built the first laser equipment with a ruby crystal stimulated by a flash lamp and mirrors to amplify the action of the laser. The beam had a wavelength of $694 \mathrm{~nm}$ and was deep red in colour. In 1962, laser was used in dentistry for the first time by Goldman. Since 1962, lasers have been used for soft tissue surgery of oral cavity with significant advantages compared to other treatment modalities. The purpose of this paper is to review the different types of lasers, laser-tissue interaction and the management of various intraoral soft tissue lesions.
\end{abstract}

Keywords:-Carbon dioxide laser,laser, Nd:YAG laser,selective absorption, soft tissue

\section{Introduction}

Laser is a special light source that has a higher power and better quality of beam compared to the other light sources. There are certain features inherent to each type of laser such as the spot size, wavelength, or radiance that is important for the kind of application intended[1,2]. The most common lasers used in surgery emit wavelengths in the infrared part of the spectrum: Nd:YAG $(\lambda=1,064 \mathrm{~nm})$, Er:YAG $(\lambda=2.94 \mu \mathrm{m})$, and CO2 laser $(\lambda=10.6$ and $9.6 \mu \mathrm{m})$.These lasers cause significant tissue heating due to the thermal energy produced, causing denaturation of proteins, decomposition of tissue, micro explosion of cell water and charring. Argon lasers emit wavelengths within the visible portion of the spectrum(between 458 and $515 \mathrm{~nm}$ ), whereas excimer lasers emit wavelengths in the ultraviolet part of the spectrum. The lasers within the ultraviolet spectrum have the ability to ionize tissues. Diode lasers emit wavelengths of 670 to $1551 \mathrm{~nm}[2,4,7,9,10,11]$

\section{Types Of Lasers}

Carbon Dioxide lasers:This is most commonly used in soft tissue surgeries. It has a wavelength of $10,600 \mathrm{~nm}$ and is readily absorbed by water. Therefore it does not penetrate too deep into the tissues $(0.1$ $0.23 \mathrm{~mm}$ ) without repeated or prolonged use. This is used ideally for superficial lesions, resurfacing of the skin and removal of sialoliths.

Nd:YAG lasers:This is mostly used in hair removal procedures. In addition, it is also used in removing tattoos and certain pigmented lesions. It has a wavelength of $1064 \mathrm{~nm}$ and is frequently used in cartilage and bone ablation.

Ho:YAG lasers:This is used for adhesions and foreign body removal while treating joint irregularities. It has a wavelength of $2.12 \mu \mathrm{m}$. This is also used in bone and cartilage ablation.

Er:YAG lasers: These lasers are most commonly used for the treatment of hard tissues, teeth and bones. They have a wavelength of $2.94 \mu \mathrm{m}$.

Alexandrite lasers:These lasers can also be used in hair removal and tattoo removal. They have a wavelength of 720-800nm.

Argon lasers $(488,514 \mathrm{~nm})$ and Krypton lasers $(531 \mathrm{~nm})$ are readily absorbed by haemoglobin and melanin and are useful in the treatment of port-wine stains.

Argon,KTP,Nd:YAG and Diode lasersare used to treat oral soft tissue lesions[3-6]

\section{Laser-Tissue Interaction}

This is mainly determined by the laser exposure time on the tissue and the effective power density depending upon the absorption of the tissue[7]. For medical applications, the most important properties when considering the interaction of the laser with tissue, are monochromaticity and high optical power[8]. If a laser beam can be transmitted through a tissue, there will be little or no absorption and therefore there will be very minimal or no thermal effects. The depth of transmission depends upon the type of tissue and the wavelength of the laser. If a laser beam cannot be transmitted through a tissue, there will be absorption, thus producing heat 
energy which can cause thermal damage to the tissue. "Selective absorption" is the key to most of the laser treatments. This is done by choosing a particular wavelength that will be selectively absorbed by the target tissue, leaving the surrounding structures unaffected[3,8]. Each tissue has specific absorption characteristics based on its composition and choromophores present(haemoglobin, water, melanin and protein). 'Cromophore' refers to the substance that is present in the target tissue that attracts the laser photon. To target a specific tissue, the selected wavelength should be strongly absorbed by the chromophores present in the particular tissue. This absorption within a target tissue's chromophore is the basis for the efficacy of the laser treatment and is referred to as laser-tissue interaction.

\subsection{Frictional keratosis}

\section{Laser Management Of Intraoral Soft Tissue Lesions}

Chronic mechanical irritation can produce a white lesion with roughened keratotic surface known as frictional keratosis. This lesion is clinically similar to leukoplakia. These lesions are reversible after the elimination of the trauma. It is usually seen in areas subjected to repeated oral trauma such as lips, buccal mucosa, lateral borders of tongue, crest of the edentulous alveolar ridge due to denture trauma. These lesions can be treated with laser therapy. Small questionable lesions can be excised by using carbon dioxide laser with a $0.2 \mathrm{~mm}$ spot size. It is applied perpendicular to the elliptical outline around the lesion. After the outline is created, the edge of the tissue is lifted using tissue forceps and the underlying tissues are dissected with laser beam at a slight angle. The lesion can be removed easily and sent for pathological examination. $[12,13]$

\subsection{Smokeless tobacco induced white lesions}

These lesions are induced by the chronic usage of smokeless tobacco. It appears as a characteristic white plaque on the mucosa in direct contact with the tobacco. It is soft and velvety on palpation and the mucosa appears wrinkled. The development of this lesion depends on the habit duration and the brand of the tobacco being used. These lesions are reversible after cessation of the habit. The lesions that persist even after cessation, especially those that exhibit ulceration, should be sent for examination. They can be excised by using the laser in a focused mode. They are usually accessible to the laser occurring in the mucolabial or mucobuccal fold in the mandible. $[12,13]$

\subsection{Nicotinic Stomatitis}

This is a form of keratosis that is associated with pipe smoking or cigar smoking. The lesion appears as a collection of red dots surrounded by a halo of white keratin. These red dots are due to the ductal openings of the minor salivary glands in response to the inflammation. These lesions are usually asymptomatic. If the patient complains of pain, burning or ulceration, laser treatment can be done to eradicate it. The lesions are vaporized after multiple punch biopsies have been taken. A carbon dioxide laser is used in a defocussed, continuous mode perpendicular to the tissue surface along the long axis if the lesion. The lesion is wiped with saline to remove the lased surface so that the nonlased surfaces will be revealed. After finishing the process, the final lased surface layer is left undisturbed to act as barrier and help in the protection of the healing surface. A palatal splint is fabricated to help the patient protect the lased surfaces during eating and drinking. An Nd:YAG contact round surgical probe can also be used in a similar manner to the carbon dioxide laser.[13]

\subsection{Solar Cheilitis}

It is a premalignant lesion involving the vermilion border of the lips and mostly the lower lips. This may be due to prolonged exposure to sunlight. Clinically the lesion appears pale and silvery gray with wrinkling. The wrinkling later becomes deep fissures and ulcerations which do not tend to bleed, although a superficial exudative crust may be present. If the lesion is not treated, it may transform into a squamous cell carcinoma. Using laser under microscopic control has been found to effective in the removal of the lesion. A carbon dioxide laser can be used at a focused spot to outline the lesion by passing between the vermilion and the hair bearing skin of the anterior lip surface and extending it towards the commissures and posteriorly to the labial vestibule. It should include about $2-3 \mathrm{~mm}$ of the surrounding normal mucosa within the target tissue. After the outline is done, the laser is held 4-6- $\mathrm{cm}$ away from the tissue and a crosshatching patter is produced in the defocussed mode. After the lasing procedure is finished, the surfaces are wiped with a moistened gauze sponge, then the surface is coated with an antibiotic dressing and a nonadherent pad is taped.[13,14]

\subsection{Leukoplakia}

Leukoplakia is defined by WHO as 'a white patch or plaque that cannot be characterized clinically or pathologically as any other disease'. It is the most common potentially malignant lesion of the oral mucosa. This is may be due to prolonged use of tobacco and alcohol, ultraviolent radiation or trauma. It usually affects persons older than 40 years of age. It most commonly affects the lips, buccal mucosa, tongue, gingiva and floor 
of the mouth. Initially they appear as grey-white plaques with demarcated borders. For smokers who do not stop the habit, the lesion extends laterally and becomes thicker. This affected mucosa becomes leathery on palpation. Some lesions have blunt projections on the surface. Hence these lesions are called verrucous leukoplakia. These lesions can be removed with laser and encourages regeneration of new, healthy epithelium. Small lesions can be removed with a focused carbon dioxide laser with a margin of 3-4mm. The decision of whether excision or vaporization should be done is based on the texture and thickness of the lesion. Thickened hyperkeratotic lesions have less water content, therefore, vaporization cannot be done. Diffuse lesions cannot be managed by excision. In such lesions, carbon dioxide lasers can be used in a defocussed mode to produce cross hatched pattern. The disadvantage of vaporization is that, a specimen cannot be taken and sent for pathological examination, so, the histology of the lesion cannot be determined.[12,13]

\subsection{Erythroplakia}

Erythroplakia is defined as a red patch that cannot be clinically or pathologically diagnosed as any other condition. It is predominant among the middle aged people. The floor of the mouth, tongue and soft palate are the most common sites of involvement. Clinically the mucosa appears as a well demarcated erythematous macule with a velvety texture. It is observed that about $50 \%$ of these lesions turn into squamous cell carcinoma. Erythroplakia is managed by excision with the help of a carbon diode laser in a pulsed mode to outline the lesion. Then the laser beam is set on focused mode in a continuous wave and a laser moat is created around the lesion. The dysplastic changes that occur in the epithelium may affect the deeper tissues also. Therefore the upper portion of the lamina propria should also be included in the specimen. Multiple deep biopsy samples should be taken to prevent infiltration.[12,13]

\subsection{Keratoacanthoma}

This is also known as self-healing carcinoma. It has a strong clinical and pathological similarity to squamous cell carcinoma. It is mostly found on the outer edge of the vermilion border of the lips. Clinically keratoacanthoma appears as a firm, sessile, dome shaped nodule. The lesion is often painful and regional lymphadenopathy may be present. The lesion can be excised with carbon dioxide laser. This is done by limited scarring of the tissue. The laser is set on pulsed mode and an outline is created around the lesion. Then the laser beam is directed perpendicular to the outline margin and a full thickness wedge is removed. Once it is removed, the tissues can be approximated with sutures.[12,13]

\subsection{Verrucous carcinoma}

It is a variant of squamous cell carcinoma characterized by exophytic growth of keratinized epithelium. It is predominant in elderly persons,60-70 years of age. It most often affects the buccal mucosa, gingiva and alveolar ridge. It is exophytic and papillary in nature. These lesions have rugae like folds with deep clefts between them. Regional lymphadenopathy may be present. The exophytic lesion can be readily excised along with the base of the lesion using a carbon dioxide laser or Nd:YAG contact laser. Whether or not laser holds a long term value in the control of leukoplakia has not been determined yet. Horsch et al reported $78 \%$ cure rate using carbon dioxide laser and hand piece. Using laser under microscopic control provided better control and precision[34].

\subsection{Oral Papillomatosis}

This is reactive tissue growth that usually develops due to ill-fitting dentures, poor denture hygiene or due to wearing the denture 24 hours a day. It usually occurs on the hard palate beneath the denture base. It is usually asymptomatic. The mucosa is erythematous and has a papillary surface. It can be managed with a carbon dioxide laser or an Nd:YAG contact laser. Sachs and Borden were the first to treat this lesion with a carbon dioxide laser[35]. Diffuse lesions can be managed by vaporization with $\mathrm{CO}_{2}$ laser after selective punch biopsies have been taken. The laser is set on continuous defocussed mode and using the cross hatching method, the area of the lesion is covered. The lased tissue surface is wiped off with saline soaked sponge. The contact Nd:YAG laser with a round probe can also be used to eliminate the lesion by stroking the surface in a similar cross hatched manner without lifting the tip of the probe from the surface of the lesion.[13]

\subsection{Lichen Planus}

Lichen planus is a common dermatologic disease that often affects oral mucosa. It usually affects the middle aged adults. The skin lesions of lichen planus have been described as purple, pruritic papules. The oral lesions are exhibited in two forms: reticular and erosive. The reticular form is characterized by interlacing white lines called Wickham's striae. The erosive form appears as erythematous area with central ulceration. Erosive lichen planus can be controlled by laser treatment. Carbon dioxide laser should be used along with selected local and systemic medications. This laser is set on a continuous, defocused mode and the usual cross hatched pattern 
is used. The contact Nd:YAG laser with round probe can also be used. This condition cannot be cured by laser treatment, it is used for palliative treatment. It has been reported from patients that there is a significant decrease in burning sensation from the lesion. Hong-Sai reported that there is an improvement in the histologic appearance after laser therapy.[13]

\subsection{Recurrent Aphthous Ulcers}

Recurrent aphthous ulcers are also known as canker sores. It is the most common oral ulcerative lesion. The exact cause of these ulcers is unknown. They can various possible etiologic factors like trauma, stress, lack of sleep, vitamin B12 deficiency, iron deficiency or immune reactions. These lesions are usually found on the lips, buccal mucosa, tongue, soft palate and floor of the mouth. Clinically they appear as a yellowish white pseudo membrane surrounded by erythematous halo. The ulcer is painful on palpation. Recently Low Level Laser Therapy(LLLT) has been used. It helps in immediate pain relief and accelerates wound healing. According to De Souza et al, $75 \%$ of the patients reported that there is a significant pain relief in the same session after laser treatment and the lesion is totally regressed in 4 days. When steroids are used, it takes 5-7 days for regression[15]. Bladowski et al also found that diode laser used at low levels reduces the wound healing period to half compared to pharmaceutical method[16]. Dr. L.J Walsh conducted study on the mechanism of action of LLLT and he concluded that LLLT reduces pain and accelerates wound healing by perhaps 'stimulating oxidative phosphorylation in mitochondria and modulating inflammatory responses' $[17,18]$. It is best to treat aphthous ulcers within the first 48 hours. A 400 micron tip is used for small lesions or an LLLT $(8 \mathrm{~mm})$ is used for larger lesions. The laser is started in a defocussed mode $5-8 \mathrm{~mm}$ away from the lesion and advanced towards the periphery $2-3 \mathrm{~mm}$ away. Continual movement from the periphery to the centre is done. A period of 15-20 seconds is given between the laser 'passes' allowing the tissue to cool. The area is rubbed with a wet gloved finger to determine if there is reduced pain felt. Likewise a $2^{\text {nd }}$ and $3^{\text {rd }}$ pass need to be done to completely reduce the pain. After each pass, the area should be palpated to check for reduced pain. It is believed that this laser treated area is less likely to reappear with a new lesion, if treated 1-2 times.

\subsection{Recurrent Herpetic Simplex Lesions}

It is a viral disease caused by Herpes simplex virus-1. The lesions break out in the keratinized mucosa such as gingiva and hard palate. Initially they appear as a collection of pinhead vesicles, later, they enlarge to form ulcerations covered by yellowish fibrin. Various lasers wavelengths have been found to be effective in treating these lesions including HeNe laser(660nm)and Erbium:YAG laser(2940nm)[19,20]. Alfonso et al looked at 232 patients using an AlGaAs diode laser in the prodromal stage, in the stage of vesicles, in the crust stage and in lesions infected secondarily. In addition the laser radiation was also applied on the vertebrae $\mathrm{C} 2-\mathrm{C} 3$ where the resident ganglion of the virus is located. It was found that $100 \%$ of the prodromal stages, $95 \%$ of the vesicular stages, $91 \%$ of the crust stages were able to cure within the first 48 hours. The patients with secondarily infected lesions needed more than 48 hours to cure, although not more than 5 days[21]. Schindl and Neumann did a study on LLLT for delaying the recurrence of the lesion outbreaks. It was found that the recurrence free interval in the laser treated group was 37.5 weeks and in the placebo group, it was 3 weeks[19].

\subsection{Mucocele}

Mucocele is a common lesion of the oral mucosa that occurs due to rupture of the salivary gland duct and spillage of the mucin into the surrounding tissues. The spillage is often due to local trauma. It is common among children and young adults. The lower lip is the most common site of involvement. Clinically it appears as a dome shaped mucosal swelling with a bluish translucent hue. The lesion often bursts, leaving a shallow painful ulcer that heals within a few days[12]. Laser treatment can be done to remove mucoceles. The mucocele can be unroofed and then excised with the gland tissue using Laser HF[13]. This laser uses high frequency technology which helps in precision cutting and reduces the risk of necrosis. The lesion is excised using fibroma removal mode $(975 \mathrm{~nm}$, continuous wave). The wound margins are sealed with a defocussed beam without side effects or complications. Re-epithelialization takes about three weeks[13].

\section{Advantages Of Laser Treatment}

- Less thermal necrosis of adjacent tissues is produced with lasers compared to electrosurgical instruments[22,23].

- During soft tissue procedures, adequate haemostasis was produced without the need for sutures[24,25].

- Accelerated wound healing with decreased post- operative discomfort and reduced need for analgesics[23,25-27].

- Minimal amount of local anaesthesia required for soft tissue treatments[23,25-28]

- Reduced operator chair time observed when soft tissue procedure is completed with lasers[23,25,27]. 
- The laser has decontaminating and bactericidal properties on the tissue, therefore, there is reduced need for antibiotics[23,25-27]

- There is immediate pain relief and inflammation associated with aphthous ulcers and herpetic lesions without pharmacological intervention[25,28,29]

\section{Disadvantages Of Laser Treatment}

- It requires additional training and education for various clinical applications and types of lasers[24,27,30,31]

- High cost required to purchase equipment, implement technology and invest in required education[24,27].

- More than one laser may be needed since different wavelengths are required for various procedures[24].

- The entire dental team and the patient should be wearing the wavelength-specific eyewear consistently[22,24].

- In immunocompromised patients, when providing soft tissue treatment of viral lesions, there is a potential risk of disease transmission from laser generated aerosol[32,33].

\section{Conclusion}

Laser technology has made rapid progress over the past few decades. Because of its many advantages, it has been widely used in oral and maxillofacial surgeries. Soft-tissue laser is a state-of-the-art tool that creates predictable aesthetic results within a general dental practice. The science, ease of use and affordability makes it simple to incorporate in general practice.

\section{References}

[1]. Golnabi, H., \& Mahdieh, M. H. (2006). Trend of Laser Research Developments in Global Level. Optics \& Laser Technology, 38(2), 122-131.

[2]. Deppe, H., \& Horch, H. H. (2007). Laser Applications in Oral Surgery and Implant Dentistry. Lasers in Medical Science, 22(4), 217-221.

[3]. Shokrollahi, K., Raymond, E., \& Murison, MS. (2004). Lasers: Principles and Surgical Applications. Journal of Surgery, 2(1), 2834.

[4]. Strauss, R. A. (2000). Lasers in Oral and Maxillofacial Surgery. Dental Clinics of NorthAmerica, 44(4), 851-873

[5]. Stabholz, A., Zeltser, R., Sela, M., Peretz, B., Moshonov, J., Ziskind, D., \& Stabholz, A. (2003). The Use of Lasers in Dentistry: Principles of Operation and Clinical Applications.Compendium of Continuing Education in Dentistry, 24(12), 935-948.

[6]. Gabrić, Pandurić. D. (2010). Physical and Ultrastructural Bone Effect Comparison BetweenLaser and Surgical Drill. PhD thesis. University of Zagreb.

[7] Müller, J. G., Berlien, P., \& Scholz, C. (2006). The Medical Laser. Medical Laser Application,21(2), 99-108

[8]. Niemz, M. H. (2007). Laser-Tissue Interactions: Fundamentals and Applications (Biologicaland Medical Physics, Biomedical Engineering). Springer-Verlag: Berlin.

[9]. Maiman, T. H. (1960). Stimulated Optical Radiation in Ruby. Nature, 187-493.

[10]. Clayman, L., \& Kuo, P. (1997). Lasers in Maxillofacial Surgery and Dentistry. Thieme: New York.

[11]. Convissar, R. A. (2010). Principles and Practise of Laser Dentistry. Mosby Elsevier: St Louis.

[12]. Neville B.W, Damm D.D, Allen C.M, Bouquot J. Oral and Maxillofacial Pathology:third edition.Pg.390,398-399,388$391,397,406,454$

[13]. Catone GA, Alling CC, Laser Applications in Oral and maxillofacial surgery.Pg:98-111

[14]. Shafer G, Hine M, Barnet L. Textbook of Oral pathology, 6th edition. pg 917.

[15]. De Souza To, Martins MA, Bussadori SK, Fernandes KP, Tanji EY, Mesquita-Ferrari RA, Martins MD. Clinical evaluation of lowlevel laser treatment for recurring aphthous stomatitis. Phtomed laser Surg. 2010 Oct;28 Suppl 2:S85-8/

[16]. Bladowski M, Konarska-Choroszucha H, Choroszucha T. Com- parison of treatment results of recurrent aphthous stomatitis (RAS) with low-and high-power laser irradiation vs. pharmaceutical method (5-year study). J Oral Laser Applic 2004;4:191-209 Photomed Laser Surg. 2010 Oct;28 Suppl 2:S85-8.

[17]. Walsh LJ. The current status of low level laser therapy in dentistry. Part 1 Soft tissue applications. Australian Dent. J. 1997; 42:4. pgs. 247-54.

[18]. Walsh LJ. The current status of low level laser therapy in dentistry. Part 2 Hard tissue applications. Australian Dent. J. 1997; 42:5. pgs 302-06.

[19]. Schindl A, Neuman R. Low-intensity laser therapy is an effective treatment for recurrent herpes simplex infection. Results from a randomized double-blind placebo- controlled study. J Invest Dermatol. 1999: 113 (2): 221-223.

[20]. Bello-Silva MS, de Freitas PM, Aranha AC, Lage-Marques JL, Simoes A, de Paula Eduardo C. Low- and high-intensity lasers in the treatment of herpes simplex virus 1 infection. Photomed Laser Surg. 2010 Feb;28(1):135-9.

[21]. http://www.laser.nu/llt/lllt_editorial12.htm\#herpes (retrieved Nov.20th, 2011).

[22]. Coluzzi D. Fundamentals of dental lasers: Science and in- struments. Dent Clin North Am 2004;48(4):751-70.

[23]. Kotlow LA. Lasers in pediatric dentistry. Dent Clin North Am 2004;48(4):889-922.

[24]. Coluzzi DJ. Lasers in dentistry. Compend Contin Educ Dent 2005;26(6A Suppl):429-35.

[25]. Boj JR, Poirer C, Hernandez M, Espassa E, Espanya A. Review: Laser soft tissue treatments for paediatric dental patients. Eur Arch Paediatr Dent 2011;12(2):100-5.

[26]. Martens LC. Laser physics and review of laser applica- tions in dentistry for children. Eur Arch Paediatr Dent 2011;12(2):61-7.

[27]. Olivi G, Genovese MD, Caprioglio C. Evidence-based dentistry on laser paediatric dentistry: Review and out- look. Eur J Paediatr Dent 2009;10(1):29-40.

[28]. Convissar RA, Goldstein EE. An overview of lasers in dentistry. Gen Dent 2003;51(5):436-40.

[29]. Green J, Weiss A, Stern A. Lasers and radiofrequency de- vices in dentistry. Dent Clin North Am 2011;55(3): 585-97.

[30]. Van As G. Erbium lasers in dentistry. Dent Clin North Am 2004;48(4):1017-59. 
[31]. Olivi G, Genovese MD. Laser restorative dentistry in chil- dren and adolescents. Eur Arch Paediatr Dent 2011;12 (2):68-78.

[32]. Parker S. Laser regulation and safety in general dental practice. Br Dent J 2007;202(9):523-32.

[33]. Garden JM, O’Bannon MK, Bakus AD, Olson C. Viral disease transmitted by laser-generated plume (aerosol). Arch Dermatol 2002;138(10):1303-7.

[34]. Horch H H, Gerlach KL, Schaefer HE, et al: Erfahrungen mit der Laserbehandlung oberflachlicher Mundschleimhauterkrankungen Dtsch Z Mund-Keifer-Gesichts-Chir 7:31-35,1983

[35]. Sachs S A, Borden GE: The utilization of carbon dioxide laser in the treatment of recurrent papillomatosis: Report of a case. J Oral Surg 39:299,1981. 